\title{
A simple crystallization device for growing large protein crystals
}

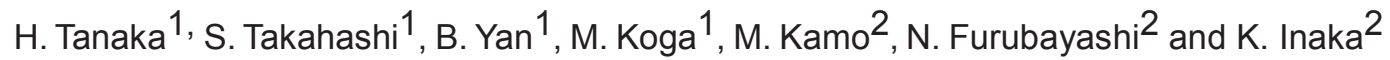

${ }^{1}$ Confocal Science Inc., 2-12-2 Iwamoto-cho, Chiyoda-ku, Tokyo 101-0032 Japan,

2Maruwa Foods and Biosciences Inc., 170-1 Tsutsui-cho, Yamatokoriyama, Nara 639-1123 Japan

The most popular method to determine three-dimensional structure of protein molecule is the $\mathrm{X}$-ray diffraction experiment. But it does not usually provide the information of the positions of hydrogen atoms in protein molecules which is important to know protonation states and hydrogen bonding networks in protein molecule. On the other hand, neutron diffraction experiment is very informative for the positions of hydrogen atoms in protein molecules ${ }^{1}$ ). But the obstruction of the neutron diffraction experiment is the requirement of much larger crystals (about $1000 \mu \mathrm{m}$ cube or beyond) if compared to the crystal size for X-ray crystallography (about $100 \mu \mathrm{m}$ cube or less).

Here we introduce a new and passive crystallization device ${ }^{2)}$ for growing large protein crystals for neutron diffraction experiment using dialysis and liquid-liquid diffusion techniques (Fig. 1). The main feature of this device is the large crystallization cell with dialysis membrane. The protein samples can be kept in the crystallization cell and only the precipitant concentration can be slowly diffused into the cell. And, if necessary, the crystallization solution can be changed during the crystallization process.

Prior to growing large crystals, we performed crystallization in the concentration range of polyethylene glycol (PEG) $4000(0-25 \%)$, sodium chloride $(0.1-1.0 \mathrm{M})$ and protein $(5-25 \mathrm{mg} / \mathrm{ml})$ using batch method to make a 3-dimensional phase diagram for optimization of crystallization condition 3 ). Lysozyme was used as a model protein. Based on the phase diagram, first we crystallized lysozyme in low PEG and low sodium chloride condition to obtain a few crystals, then we increased the sodium chloride concentration first and successively PEG in the crystallization solution to grow crystals larger with higher resolution. The large crystals obtained by this procedure are shown in Fig. 2.

We concluded that this crystallization device and the crystallization strategy for growing large crystals worked effectively. This device is simple so that it can be applicable not only for the laboratory experiment but also for the crystallization experiments in space where larger crystals are expected to grow.

\section{References}

1) J. D. Ng et al. Acta Cryst. (2015). F71(4), 358-370.

2) Patent pending

3) S. Takahashi et al. http://www.xray.cz/iccbm/files/749.htm

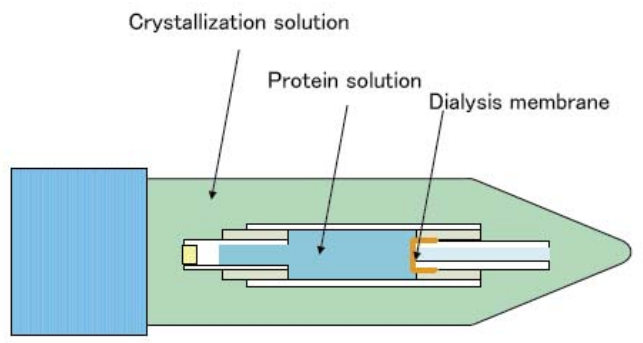

Fig. 1 Crystallization device for growing large crystals.

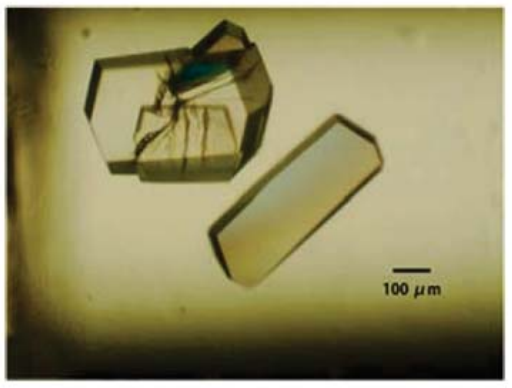

Fig. 2 Lysozyme crystals. 